\title{
Percepções de nutricionistas sobre o apoio matricial e núcleo ampliado de saúde da
}

\section{família e atenção básica}

\author{
Nutritionists' perceptions of matrix support and expanded family health and primary care \\ Percepciones de los nutricionistas sobre el apoyo de la matriz y la salud familiar ampliada y la \\ atención primaria
}

Recebido: 29/11/2021 | Revisado: 07/12/2021 | Aceito: 10/12/2021 | Publicado: 20/12/2021

\author{
Luis Rocildo Caracas Vieira e Souza \\ ORCID: https://orcid.org/0000-0002-9739-7993 \\ Universidade Estadual do Ceará, Brasil \\ E-mail: rocildo.nutri@gmail.com \\ Fátima Café Ribeiro dos Santos \\ ORCID: https://orcid.org/0000-0003-2350-6701 \\ Universidade Estadual do Ceará, Brasil \\ E-mail: fatimacoffee@gmail.com \\ Maria Marlene Marques Ávila \\ ORCID: https://orcid.org/0000-0002-8511-2524 \\ Universidade Estadual do Ceará, Brasil \\ E-mail: marlene.avila@uece.br
}

\begin{abstract}
Resumo
Uma das formas de inserção do nutricionista na atenção primária é por meio do Núcleo Ampliado de Saúde da Família e Atenção Básica (NASF-AB), assim, esse estudo buscou compreender a atuação deste profissional sob a perspectiva do apoio matricial (AM) por meio de um estudo exploratório, de abordagem qualitativa, realizado com as nutricionistas dos NASF-AB de Tauá/CE. A pesquisa de campo ocorreu em dezembro de 2018 com o recurso de entrevistas semiestruturadas. A análise ocorreu pela técnica da análise temática, que possibilitou identificar cinco categorias empíricas: conceito de NASF-AB; trabalho em equipe; (in)compreensão de AM; AM: suporte assistencial e suporte pedagógico; acionamento do profissional pela EqSF; e, desafio e potencialidades para atuação no NASF-AB. A análise evidenciou o distanciamento entre pressupostos teórico-práticos centrais e o processo de trabalho, contribuindo para a fragmentação do cuidado, distanciamento entre os profissionais, predomínio de encaminhamentos impessoais e ações de enfoque ambulatorial, entrave para a interprofissionalidade e interdisciplinaridade, condições essenciais para a integralidade em saúde. Como sugestão, a realização de ações de educação permanente com base no AM e seus pressupostos.
\end{abstract}

Palavras-chave: Atenção primária à saúde; Nutricionistas; Integralidade em saúde; Pesquisa qualitativa.

\begin{abstract}
One of the ways of insertion of the nutritionist in primary care is through the Expanded Center for Family Health and Primary Care (NASF-AB), thus, this study sought to understand the performance of this professional from the perspective of matrix support (MS) through of an exploratory study, with a qualitative approach, carried out with the nutritionists of the NASF-AB in Tauá/CE. The field research took place in December 2018 using semi-structured interviews. The analysis was carried out using the thematic analysis technique, which made it possible to identify five empirical categories: concept of NASF-AB; team work; incomprehension of MS; MS: care support and pedagogical support; activation of the professional by EqSF; and, challenge and potential for acting at NASF-AB. The analysis showed the gap between central theoretical-practical assumptions and the work process, contributing to the fragmentation of care, distance between professionals, predominance of impersonal referrals and outpatient focused actions, obstacles to interprofessionality and interdisciplinarity, essential conditions for comprehensiveness in health. As a suggestion, carrying out permanent education actions based on MS and its assumptions.
\end{abstract}

Keywords: Primary health care; Nutritionists; Integrality in health; Qualitative research.

\section{Resumen}

Una de las formas de inserción del nutricionista en la atención primaria es a través del Centro Ampliado de Salud de la Familia y Atención Primaria (NASF-AB), por lo que este estudio buscó comprender el desempeño de este profesional desde la perspectiva de la matriz de apoyo (MA). a través de un estudio exploratorio, con enfoque cualitativo, realizado con los nutricionistas de la NASF-AB en Tauá / CE. La investigación de campo se llevó a cabo en diciembre de 2018 mediante entrevistas semiestructuradas. El análisis se realizó mediante la técnica de análisis 
temático, que permitió identificar cinco categorías empíricas: concepto de NASF-AB; trabajo en equipo; (mal)entendido de MA; MA: apoyo asistencial y apoyo pedagógico; activación del profesional por EqSF; y desafío y potencial para actuar en NASF-AB. El análisis mostró la brecha entre los supuestos teórico-prácticos centrales y el proceso de trabajo, contribuyendo a la fragmentación de la atención, distanciamiento entre profesionales, predominio de derivaciones impersonales y acciones ambulatorias, obstáculos a la interprofesionalidad e interdisciplinariedad, condiciones esenciales para la integralidad en salud. Como sugerencia, realizar acciones de educación permanente basadas en MA y sus supuestos.

Palabras clave: Atención primaria de salud; Nutricionistas; Integralidad en salud; Investigación cualitativa.

\section{Introdução}

A atuação em equipe multiprofissional, por meio do compartilhamento de saberes e práticas, objetivando a integralidade da atenção é pressuposto essencial para a atuação interprofissional que deve caracterizar o trabalho realizado pelo Núcleo Ampliado de Saúde da Família e Atenção Básica (NASF-AB). Nesse sentido, a tecnologia que norteia a atuação do profissional do NASF-AB é o apoio matricial (AM) (Brasil, 2017a, 2014).

Na Atenção Primária à Saúde (APS), o AM configura-se como arranjo organizacional para o compartilhamento de saberes e práticas, reduzindo a fragmentação da atenção e promovendo a constituição de uma rede de cuidados que aumente a qualidade e a resolutividade das ações em saúde. Nesse contexto, o AM é recomendado como estratégia para integração entre Equipes de Saúde da Família (EqSF) e NASF-AB, revelando-se como proposta democrática e dialógica, baseada no estabelecimento de relacionamentos horizontais entre os diferentes trabalhadores de saúde, divergindo do modelo hegemônico marcado pelo enfoque ambulatorial (Souza, 2018).

A oferta de retaguarda assistencial especializada - assistência clínica dispensada aos usuários, e o suporte pedagógico à EqSF apoiada por meio de espaços de educação permanente são as duas dimensões, ou eixos, que orientam o trabalho do AM. A execução dessa tecnologia depende da construção compartilhada de diretrizes clínicas e sanitárias (Viana \& Campos, 2018).

Com a criação do Núcleo de Apoio à Saúde da família (NASF), através da Portaria GM nº 154, de 24 de janeiro de 2008, profissionais de diferentes áreas do conhecimento foram incorporadas na APS, entre elas o nutricionista. A inserção deste profissional se dá pela necessidade de desenvolver estratégias de educação permanente e organização dos processos de trabalho da EqSF, ampliando a capacidade desses profissionais no controle e prevenção dos distúrbios nutricionais existentes no território de atuação das equipes de saúde (Brasil, 2017b, 2018).

O aprofundamento de reflexões acerca de como o AM influencia na prática do nutricionista é essencial para qualificar sua atuação na APS e verificar o impacto que essa tecnologia de trabalho proposta pelo Ministério da Saúde tem produzido nos serviços de saúde apoiados pelos NASF-AB.

Todavia, embora o AM direcione para um cuidado a partir de práticas dialógicas de cuidado colaborativo, estudos recentes têm apontado dificuldades quanto a sua compreensão e operacionalização na APS (Souza et al., 2017; Castro \& Campos, 2016; Castro et al., 2016; Oliveira \& Campos, 2017; Klein \& d'Oliveira, 2017; Hirdes \& Oliveira 2017).

Diante do exposto, esse estudo teve como objetivo compreender a atuação do nutricionista no NASF-AB a partir dos marcos teóricos-conceituais e metodológicos do AM.

\section{Metodologia}

Trata-se de um estudo exploratório e de abordagem qualitativa. O caráter exploratório diz respeito à intenção de ampliar o conhecimento acerca de um tema de inserção recente em saúde pública. A abordagem qualitativa lida com o universo dos significados, atitudes, crenças e valores, abrangendo áreas mais profundas das relações, processos e fenômenos sociais (Gil, 2008; Minayo, 2006). 
O cenário da pesquisa foi o município de Tauá/CE, situado na região dos Inhamuns, a $340 \mathrm{~km}$ de Fortaleza. É o segundo maior município em extensão territorial do Ceará, com 4.018,162 km² de território e estimativa de 58.517 habitantes (Instituto Brasileiro de Geografia e Estatística, 2019). O município foi escolhido como campo da pesquisa por ser uma das cidades cearenses pioneiras na implantação dos NASF-AB em 2008, contando com cinco equipes de apoio instaladas, todas com nutricionistas; a APS está estruturada com vinte e cinco EqSF e dezenove unidades de saúde da família (USF) distribuídas em 5 regiões de saúde (I, II, III, IV e V), ofertando 100\% de cobertura populacional; além de ser o cenário de prática do pesquisador enquanto residente no Programa de Residência Multiprofissional em Saúde pela Escola de Saúde Pública do Ceará.

As informantes foram cinco nutricionistas dos NASF-AB de Tauá/CE. Os dados foram construídos por meio de entrevista individual norteada por roteiro semiestruturado abrangendo características sociodemográficas das informantes, concepçães sobre NASF-AB e atuação profissional. A construção dos dados a partir das entrevistas ocorreu durante o mês de dezembro de 2018 e janeiro de 2019, nas USF onde os informantes atuavam, em sala reservada, conduzida pelo pesquisador responsável e com duração média de 35 minutos. A forma de registro foi a gravação em áudio.

Os dados foram analisados pela técnica de análise temática (AT), derivada da análise de conteúdo de Bardin. A AT consiste em descobrir os núcleos de sentido na comunicação, sendo desenvolvida em três etapas: pré-análise, envolvendo leituras exaustivas das transcrições das entrevistas; exploração do material, sintetizando os resultados em categorias temáticas; e interpretação à luz de referenciais teóricos pertinentes (Bardin, 2016).

$\mathrm{Na}$ demonstração dos resultados, as identidades das informantes foram preservadas identificando os depoimentos pelo código: N1, N2, N3, N4, N5.

O estudo cumpriu as exigências éticas da resolução 466/2012 (Brasil, 2012), com anuência da Secretaria Municipal de Saúde de Tauá e aprovação pelo Comitê de Ética em Pesquisa da Escola de Saúde Pública do Ceará, sob Parecer no 3.059.607.

\section{Resultados e Discussão}

Inicialmente encontra-se a caracterização sociodemográfica das informantes. Em seguida, as categorias empíricas: conceito de NASF-AB; trabalho em equipe; (in)compreensão de AM; AM: suporte assistencial e suporte pedagógico; acionamento do profissional pela EqSF; desafios e potencialidades para atuação no NASF-AB.

\section{Caracterização sociodemográfica}

As informantes eram do sexo feminino, na faixa etária de 20-30 anos. Quanto a qualificação profissional, quatro tinham pós-graduação lato sensu. Quanto ao tempo de atuação no NASF-AB, uma informante atuava há mais de seis anos e as demais tinham menos de três anos.

Todas foram admitidas para o regime de trabalho de 40 horas semanais, por contratação temporária, configurando um precário vínculo de trabalho. Quanto ao número de equipes apoiadas, três profissionais apoiavam cinco EqSF, uma apoiava quatro e outra seis.

\section{Conceito de NASF-AB}

Essa categoria emergiu nas falas das entrevistadas após serem questionadas sobre o que seria o NASF-AB para elas. É pertinente aqui destacar que as nutricionistas ainda utilizavam a antiga nomenclatura de Núcleo de Apoio à Saúde da Família (NASF).

"O NASF pra mim é o núcleo de apoio à saúde da família. Eu acho que é essencial o NASF, a existência, porque eu 
acho que se completa, né? É uma equipe multiprofissional.” N1

"Núcleo de apoio à saúde da família [risos]. É um órgão que atua como equipe multidisciplinar, que acho que visa na melhoria da qualidade de vida do paciente [...]." N2

A referência ao NASF-AB como sendo uma equipe multiprofissional alinha-se com o conceito proposto pelo Ministério da Saúde que caracteriza o NASF-AB como equipes formadas por diferentes categorias profissionais orientadas pela atuação integrada e apoiando as EqSF e outras modalidades de equipes de atenção básica (Brasil, 2014).

Atuar de forma integrada pressupõe que não basta apenas uma equipe possuir profissionais de diferentes categorias, uma vez que a atuação isolada dos profissionais pode contribuir para a fragmentação do cuidado, com enfoque na doença e não no indivíduo-família-comunidade. É necessário que a atuação ocorra de maneira dialógica e pactuada, na perspectiva da interdisciplinaridade, aqui entendida enquanto interação comunicativa entre diferentes conhecimentos (Aciole \& Oliveira, 2017).

Destaca-se a presença de expressões como "suporte" e "apoio" nos relatos das informantes, evidenciando que associavam ao NASF-AB uma atuação complementar às EqSF, como na fala a seguir:

"Assim, a gente ajudar, na questão da ESF pra dar um suporte maior, ter todo esse suporte que eles já precisavam e graças a Deus implementaram o NASF pra gente estar ajudando [...]." N3

"Pelo nome a gente já sabe que é um apoio à estratégia (de saúde da família)." N4

Percebe-se nas falas a ideia do NASF-AB enquanto proposta que visa gerar impactos positivos na APS junto às EqSF. Esse entendimento harmoniza-se com o contexto da criação do NASF-AB, que teve como objetivo apoiar, ampliar, aperfeiçoar a atenção e a gestão da saúde na APS (Brasil, 2014).

A presença de expressões como "suporte", "estar ajudando" e "apoio" pressupõe que as informantes ofertam algum tipo de apoio especializado. Entretanto, tal apoio não deve ser visto como ambulatório de especialidades dentro da APS, uma vez que a atuação do profissional no NASF-AB implica no compartilhamento de saberes e práticas, objetivando tanto ofertar cuidado integral ao paciente como ampliar a capacidade clínica dos profissionais das EqSF apoiadas (Brasil, 2014; Fittipaldi et al., 2017).

Em uma contextualização histórica, com a aprovação e publicação da nova Política Nacional de Atenção Básica (PNAB) no ano de 2017, o NASF passou a ser chamado de NASF-AB, retirando o termo "apoio" da nomenclatura (e do texto específico da política que trata do NASF-AB) que foi substituído por "ampliado", enfatizando que a atuação do NASF-AB contempla outros formatos de equipes que não pertençam à modalidade de saúde da família (ESF) (Brasil, 2017a). Segundo Viana e Campos (2018), a dimensionalidade do "apoio", termo oriundo do método paidéia, aponta para a construção de espaços de cogestão do cuidado e da capacidade de somar conhecimentos de diferentes núcleos de saber, elementos fundamentais para os processos de trabalho no NASF-AB.

Quando o Ministério da Saúde criou em 2008 o Núcleo de Apoio à Saúde da Família, normatizou que a dimensão do apoio, presente na sua nomenclatura, seria elemento direcionador no esforço de reestruturação do processo de trabalho em saúde (Brasil, 2008, 2014). Tal reestruturação não deveria ser alcançada apenas pela incorporação de novas categorias profissionais, uma vez que "multiprofissional" não significa necessariamente "cuidado compartilhado", podendo até intensificar a fragmentação (já presente) na assistência, mas por uma lógica de cuidado pautado pela integralidade, interdisciplinaridade, de maneira democrática e pelo trabalho em equipe horizontal (Nascimento \& Cordeiro, 2019). 


\section{Trabalho em equipe}

Questionadas a respeito de como se dá o trabalho em equipe, principalmente sobre a relação entre NASF-AB e EqSF, as informantes relataram a existência de entraves que prejudicavam a atuação integrada.

"São bem dispersos, afastados. Acho que porque a referência (EqSF) é mais ambulatório e a porcentagem maior tem que ser educação em saúde. Tem que ter educação em saúde, mas quando precisa ser junto, eles (EqsF) tem resistência [...].” N2

O distanciamento entre os profissionais da APS, a exemplo do relatado acima, é um dos fatores responsáveis pela reprodução do paradigma biomédico, centrado na doença, com foco em procedimentos e na medicalização do cuidado em saúde. A integralidade no cuidado, considerada a principal diretriz a ser praticada pelo NASF-AB, só é possível por meio do trabalho interdisciplinar em equipes integradas, de forma a desenvolver práticas colaborativas e interprofissionais (Santos et al., 2018).

Atuar em equipe exige um processo contínuo de construção de processos de trabalho baseados na lógica do compartilhamento e da corresponsabilização do cuidado. Dentre as dificuldades existentes para a atuação com foco na integralidade é possível citar: priorização de atendimentos ambulatoriais, desenvolvimento de práticas assistenciais em detrimento das ações de promoção e prevenção, resistência dos profissionais em modificar suas práticas e dificuldades relacionadas ao modelo formador dos cursos da área da saúde que não preparam os futuros profissionais para o trabalho em equipe (Fittipaldi et al., 2017; Cynthia et al., 2018; Faria et al., 2018 Spina et al., 2018).

Além disso, a organização dos processos de trabalho dificulta a integração entre os profissionais, por exemplo, a impossibilidade de participar de reuniões de equipe foi apontada como fragilidade para a construção do vínculo e compartilhamento do cuidado entre profissionais.

\footnotetext{
"Se eu participasse de todas as reuniões de equipe eu tinha maior vínculo com as equipes, (saberia) dos problemas que acontecem. Mas não participo porque em cada reunião estou em um lugar diferente. [...] Acho que o trabalho em equipe deixa a desejar." N5
}

Em estudo semelhante com nutricionistas que atuavam no NASF-AB, os autores identificaram que os encontros para reuniões eram momentos propícios para a discussão de casos acompanhados e pactuação de atividades, gerando maior integração entre os profissionais (Campos et al., 2017). Achados semelhantes foram encontrados em pesquisa realizada em Sobral - CE (Neto et al., 2018).

Nesse sentido, o apoio e incentivo dos gestores são fundamentais para que os profissionais de saúde pactuem e participem periodicamente de reuniões de equipe, uma vez que estas são consideradas espaços coletivos essenciais para o desenvolvimento da gestão compartilhada do cuidado, condição essencial para a execução de ações interdisciplinares (Spina et al., 2018).

\section{(In)compreensão a respeito do AM}

As informantes expressaram incompreensão quanto ao $\mathrm{AM}$, pressuposto essencial ao trabalho do NASF-AB, conforme demonstram os depoimentos abaixo:

"Já ouvi falar, mas não sei como funciona direito. Não sei. É uma dificuldade que eu tenho: apoio matricial. Já ouvi falar sobre matriciamento." N1 
"Não. Não tenho ideia do que seja.” N3

“Já ouvi, mas não saberia dizer. Se li, não lembro muito.” N5

Resultado semelhante foi revelado por pesquisa realizada em Florianópolis que avaliou a atuação dos profissionais do NASF-AB, e apontou que a maioria possuía conhecimento insatisfatório sobre o AM, contribuindo para uma menor integração entre os profissionais do NASF-AB e as equipes apoiadas (Souza et al., 2017).

Conhecer o significado de AM e como se dá a sua incorporação nos processos de trabalho entre NASF-AB e EqSF são fatores essenciais para arranjos organizacionais e relações de trabalho que propiciem a mudança da atenção centrada da categoria profissional para o compartilhamento entre a equipe, do cuidado fragmentado para a integralidade da atenção, do foco na doença para o indivíduo-família-comunidade (Viana \& Campos, 2018), diferencial do trabalho interprofissional, centrado no usuário.

O AM é formado por dois conceitos operadores: "apoio" e "matricial". O "apoio" refere-se aos processos de trabalho entre profissional de referência e apoiador/especialista, não com base em autoridade/relações de poder, mas na construção de conceitos dialógicos. Nesse sentido, o profissional apoiador constrói, de maneira compartilhada, propostas de intervenção, contribuindo com seu núcleo de saber, experiências e visão de mundo, articulando-as com demandas trazidas pelos sujeitos implicados no processo de cuidado. O conceito "matricial" representa a superação da lógica de relações verticais, hierarquizadas e de transferência de responsabilidades, tradicionais em sistemas de saúde, para a lógica de relacionamentos horizontais e de produção de intervenções terapêuticas integradas (Viana \& Campos, 2018; Castro \& Campos, 2016; Castro et al., 2016; Oliveira \& Campos, 2017).

Cabe destacar que o $\mathrm{AM}$, nos seus eixos assistencial (cuidados dispensados à população) e pedagógico (incorporação de saberes e práticas aos profissionais das equipes apoiadas), inspirou o projeto e a implantação do NASF-AB (Brasil, 2014). Para Viana e Campos (2018), atuar como apoiador matricial significa a efetivação de arranjos de cuidado compartilhado e elevação da resolutividade da APS.

Dos depoimentos das informantes que relataram possuir alguma familiaridade com a tecnologia, sobressai a superficialidade e redução de compreensão que situa o AM ao âmbito do Centro de Atenção Psicossocial (CAPS) na rede assistencial do município.

"Nunca li. Já ouvi falar. Aquele do CAPS? Nunca fui convidada. Acho que seja equipe multidisciplinar [...]. Eu vejo pelo lado do CAPS: reúne os principais profissionais (psicólogo, terapeuta - ocupacional -, assistente social) para discutir casos clínicos, então... isso... discussão de casos clínicos para melhoria do paciente.” N2

“[....]. Li muito pouco sobre isso, não vou mentir. Mas pelo que eu vejo é como se fosse um apoio geral de todos os profissionais, não da ESF, pelo que notei. Como se fosse o CAPS se juntando com a ESF. É esse o apoio matricial que você está falando?" N4

As falas evidenciam a incompreensão das informantes quanto ao significado do AM e suas implicações para seus processos de trabalho na APS, sendo possível depreender, no contexto analisado, a existência de profissionais de saúde que atuam desconhecendo as políticas que orientam a organização do serviço. Apesar da diversidade de publicações do Ministério da Saúde que orientam a atuação do NASF-AB (Brasil, 2014, 2017a, 2017b), além de cursos ofertados em plataformas digitais para qualificação profissional (Souza, 2018), as informantes relataram que nunca leram ou leram muito pouco sobre AM, fazendo referência ao seu uso apenas em contextos que envolvam o CAPS.

As reflexões e observações sistemáticas que contribuíram para construção das bases teóricas do AM levaram em consideração os processos de trabalho de equipes de saúde mental inseridas na APS, na cidade de Campinas - SP, na década de 
1990. Nesse sentido, a proposta do AM deriva da tentativa de articular os cuidados em saúde mental na atenção primária. A partir da criação do Núcleo de Apoio à Saúde da Família em 2008, a portaria nº 154, de janeiro de 2008 prevê o aporte financeiro para a criação e estruturação de equipes multiprofissionais orientadas pelo AM. Essa foi a primeira vez que a tecnologia esteve presente na definição de uma portaria do Departamento de Atenção Básica do Ministério da Saúde, tornandose eixo estruturante em uma política de saúde pública (Castro \& Campos, 2016; Campos et a., 2014).

Apreende-se, ademais, que os achados de incompreensões a respeito do AM no contexto analisado apontam para a necessidade de investimentos em estratégias de educação permanente, qualificando os profissionais para atuarem conforme as diretrizes propostas para o NASF-AB (Brasil, 2014, 2017a, 2017b).

\section{Apoio matricial: suporte assistencial e suporte pedagógico}

A proposta do AM na APS envolve a percepção de que as ações do NASF-AB devem contemplar dois públicos: o usuário e as EqSF apoiadas. O suporte assistencial é contemplado pelas ações clínicas junto aos usuários - consultas individuais, atendimentos em grupos, atividades de educação em saúde, dentre outros. O suporte pedagógico refere-se ao apoio educativo realizado com os profissionais das equipes apoiadas, evidenciando nesse caso o desenvolvimento de ações compartilhadas que proporcionem a educação permanente (Brasil, 2014).

As falas a seguir evidenciam que as intervenções realizadas pelas informantes possuíam enfoque em atendimentos específicos do núcleo profissional, em detrimento de ações conjuntas com a EqSF, contribuindo assim para a fragmentação do cuidado.

"Só faço ambulatório, muito pouca atividade (educativa), pelo menos algum domiciliar na semana tem [...]. Não são frequentes (atividades compartilhadas com EqSF)" N1

"Ambulatório; [atendimento] domiciliar não tenho muitos, mas faço [...]. Já fiz ambulatório uma vez só com médico [...]. Não é rotina." N2

"Ambulatório, domiciliar, palestra em grupos, escolas. Desenvolvo mais atividades sozinha do que com outros profissionais." N4

Deve existir um equilíbrio entre as duas dimensões/suporte para que não haja a predominância de intervenções individualizadas com enfoque ambulatorial. Atuar sob a ótica do AM significa agir na perspectiva do trabalho interprofissional, por meio do trabalho compartilhado e da cogestão do cuidado (Castro \& Campos, 2016).

Quando questionadas a respeito das dificuldades de compartilhar o cuidado com os demais profissionais, as informantes deram as seguintes respostas:

"Incompatibilidade de agenda é o maior problema de fazer atendimento compartilhado com médico e enfermeira, um dia é visita da equipe de referência, aí estou em outro território, ou então é porque é dia de pré-natal e não tem como dar certo." N1

"É o desafio do NASF: saber e poder compartilhar. [Existe] pouco interesse dos colegas de compartilhar conhecimento. Muita vaidade de compartilhar. Focam muito no quantitativo e não qualitativo.” N2

Estudos apontam possíveis entraves que dificultam a atuação dos profissionais sob a lógica do AM, tais como: resistências dos profissionais, desconhecimento ou má compreensão sobre a tecnologia, forte apego ao modelo biomédico, modelos de gestão baseados em metas de produção, precarização do vínculo profissional com o serviço, incompatibilidade de agendas profissionais e excesso de demandas na APS (Castro \& Campos, 2016; Castro et al., 2016; Oliveira \& Campos, 2017; Klein \& d'Oliveira, 2017; Hirdes \& Oliveira, 2017). 
Como estratégias para superação da fragmentação do cuidado, passando pela reorganização da atenção dispensada pelas equipes, a literatura especializada sugere: apoio da gestão para execução do AM, realização de educação permanente com os profissionais, criação de espaços de discussão e construção compartilhadas, fortalecimento do vínculo com o serviço, construção pactuada das agendas de trabalho, definição e qualificação dos protocolos de encaminhamentos, estabelecimento de avaliação do NASF-AB baseados nas competências do AM, dentre outros (Neto et al., 2018; Campos et al,. 2014).

\section{Acionamento do nutricionista pela EqSF}

O distanciamento teórico a respeito do conceito de AM, evidenciado nas falas das informantes, acarreta consequências na atuação profissional, conforme percebido pelo predomínio de intervenções individuais em detrimento de ações compartilhadas. Questionadas sobre a forma de acionamento do apoio especializado em nutrição pela EqSF, sobressaiu a centralização em encaminhamentos impessoais para a transferência do cuidado:

"A maioria das vezes já vem com encaminhamento, o papel escrito. O médico encaminha já dizendo o motivo pelo qual o paciente está sendo encaminhado [...].” N1

"Com encaminhamento de papel. Não sempre. Às vezes a enfermeira atende uma gestante com anemia e pede para (a paciente) conversar com a nutricionista. Aí eu atendo. Mas 80\% é encaminhamento [...].” N2

"Encaminhamento por papel. Encaminham e o paciente marca na recepção [...].” N4

Em estudo semelhante realizado em Santos - SP, as pesquisadoras perceberam que as nutricionistas concentravam suas atividades em atendimentos ambulatoriais, a partir do encaminhamento médico, sem que houvesse discussão entre os profissionais (Spina et al., 2018). Nesse mesmo sentido, a falta de comunicação e integração entre profissionais é percebida como um dos fatores responsáveis pela elevada utilização dos encaminhamentos impessoais (Ferrer et al., 2015).

O encaminhamento impessoal, também denominado de tradicional, constitui-se como empecilho para o compartilhamento da responsabilização clínica e sanitária, essencial para o trabalho interprofissional na APS, uma vez dificulta a criação de espaços para discussão das condutas terapêuticas instituídas no cuidado em saúde (Bispo Júnior \& Moreira, 2018; Maffissoni et al., 2018).

A utilização da ferramenta do AM é condição essencial para superar esse modelo tradicional por uma forma dinâmica e interativa, utilizando estratégias dialógicas que envolvam a ampliação de possibilidades terapêuticas. Discussão de casos e temas, pactuação de protocolos de acionamento do apoio especializado, intervenções compartilhadas e construção de projetos terapêuticos singulares (PTS) são algumas das estratégias que o NASF-AB dispõe para reorganização do cuidado (Brasil, 2014; Viana \& Campos, 2018).

\section{Desafios e potencialidades para atuação no NASF-AB}

Outra categoria que emergiu nas entrevistas foi relacionada às dificuldades e potencialidades percebidas para a atuação da categoria no NASF-AB. As dificuldades relatadas pelas informantes foram essencialmente relacionadas às condições de trabalho na APS do município:

"Material, principalmente quando tem ação do PSE (Programa Saúde na Escola), balança estadiômetro, fita métrica, material de Xerox para levar, transporte para visita. Mais salas seria muito bem-vindo. É muita gente do NASF para pouca sala [...].” N1

"Estadiômetro não tem, fita é minha, balança da ACS. Domiciliar é com meu transporte, não tem carro. Só vou se o paciente realmente necessitar [...].” N2 
“[...] A estrutura física, a questão das salas. Muito profissional do NASF de várias categorias e não tem sala. A gente tem que ficar em corredores, atender na sala de enfermeira. Se eu quiser atender não tenho como atender porque não tem sala [...].” N5

A falta de recursos, como materiais educativos, balança, estadiômetro, fita métrica, disponibilização de transporte para atendimentos domiciliares, foram referidas por todas, achado semelhante de outros estudos com integrantes do NASF-AB que relataram a precariedade de recursos físicos como obstáculo à atuação profissional (Gozzi, 2017; Gonçalves et al., 2015; Santos, 2018).

A precariedade da estrutura física e equipamentos são obstáculos consideráveis e requerem a devida atenção dos gestores públicos, contudo, cabe destacar que a atuação do profissional na APS, em particular, no NASF-AB, não deve ser limitada devido a esses entraves.

A produção do cuidado em saúde na APS deve ocorrer mediante o trabalho vivo em ato, ou seja, priorizando o uso de tecnologias leves, ferramentas presentes no encontro relacional entre profissionais de saúde e usuários, como a escuta qualificada, empatia e a construção de vínculo e confiança, além do AM, PTS e clínica ampliada, à utilização das tecnologias leve-duras e duras, representadas respectivamente pelo uso de saberes estruturados, equipamentos e materiais (Bispo Júnior \& Moreira, 2018; Merhy et al.,2016).

Quanto ao relato da falta de salas/consultórios para atendimentos, é previsto que os profissionais do NASF-AB se integrem ao cronograma de utilização dos espaços das UBS, considerando que sua atuação deve privilegiar o compartilhamento do cuidado, ao invés do modelo de ambulatório especializado (Brasil, 2014).

Contudo, há, também, potencialidades percebidas no processo de trabalho do NASF-AB, como é possível perceber nos seguintes relatos:

“A equipe multiprofissional se ajuda. É uma potencialidade. Às vezes a gente não tem algum recurso e o outro traz de casa e ajuda. A comunicação do NASF é boa, é aberta [...].” N1

"Adoro esse trabalho em equipe. Na verdade, esse trabalho do NASF é mais gratificante. É uma equipe muito junta pelo paciente, abraçam mesmo, se mobilizam, pensam muito no paciente [...]." N3

Conforme já exposto na categoria "Trabalho em equipe", havia distanciamento entre as equipes de saúde, resultando em fragmentação do cuidado na APS. Dessa forma, quando as informantes disseram que "A comunicação do NASF é boa, é aberta" e "É uma equipe muito junta pelo paciente" subentende-se que os processos de trabalho entre os profissionais que integram o NASF-AB ocorriam de maneira articulada, contrapondo-se à falta de integração em atividades que envolvessem as EqSF.

A existência do vínculo, não apenas entre os profissionais do NASF-AB, mas, também, entre equipe de apoio e EqSF é condição essencial para que ocorra o trabalho de forma que favoreça a horizontalização do cuidado, permitindo que práticas e saberes sejam agregados e compartilhados pelos integrantes da equipe. É importante destacar que a existência da equipe multiprofissional não significa que o trabalho ocorra de forma interdisciplinar e interprofissional. Interdisciplinaridade pressupõe a integração entre diferentes áreas de conhecimento, por meio de um processo dialógico e solidário, ampliando a capacidade do cuidado (Castro et al., 2016; Azevedo et al., 2017; Seixas et al., 2018), enquanto interprofissionalidade implica o compartilhamento de práticas (Costa et al., 2018).

O reconhecimento da importância do NASF-AB por parte da população esteve presente em algumas falas, evidenciando que a relação usuário-profissional tem gerado impacto positivo nos profissionais. 
“[...] O bom do NASF é ser reconhecido pela população. Por mais que não tenha o retorno do gestor, mas temos da população [...].” N5

A construção de relações que envolvam o afeto e a confiança entre usuários e profissionais do SUS, como percebido pelo relato acima, é considerado como um potencializador terapêutico, contribuindo para a construção do cuidado de forma longitudinal (Arce \& Teixeira 2017).

\section{Considerações Finais}

Como contribuições do presente estudo, destacamos as considerações relacionadas à atuação do nutricionista do NASF-AB, os entraves para sua atuação sob a perspectiva do AM e possíveis ações que viabilizam o trabalho integrado em equipe na APS no contexto analisado.

Os resultados permitem inferir que apesar das nutricionistas compreenderem o NASF-AB como equipe multiprofissional atuando em suporte para as EqSF apoiadas, centralizavam suas intervenções em atividades individuais, de caráter assistencial, sendo acionadas majoritariamente por encaminhamentos impessoais, reproduzindo uma assistência fragmentada.

O distanciamento teórico-prático da proposta do AM, conceito central para atuação no NASF-AB, evidencia a necessidade de pautar os processos de trabalho na APS no compartilhamento de saberes e práticas entre os profissionais envolvidos no cuidado. Ressaltamos que a implementação de mudanças nos processos de trabalho, historicamente cristalizados nos serviços de saúde, demanda um apoio da gestão local para a mobilização dos profissionais das equipes envolvidas e implantação de novos modelos de cuidado orientados pela diretriz da integralidade.

A realização de ações de educação permanente, baseadas no AM e seus pressupostos, é apontada como sugestão para que os gestores municipais orientem os trabalhadores da APS na reorganização do cuidado. Apesar das informantes serem da área específica da Nutrição, o estudo aponta fragilidades quanto ao AM, que não dizem respeito apenas ao profissional nutricionista, mas abrangem demais categorias profissionais integrantes do NASF-AB, o que dá ênfase à importância das ações de educação permanente.

Os resultados deste estudo dizem respeito ao contexto de saúde na APS no cenário estudado, entretanto, dados resultados semelhantes encontrados na literatura pertinente, contribuem para a compreensão de situações que podem ocorrer em outros cenários de prática no NASF-AB. São ainda necessários novos estudos que avaliem a implementação e desenvolvimento do AM no âmbito da APS, indicando o impacto dessa tecnologia nos processos de trabalho das equipes de saúde.

\section{Referências}

Aciole, G. G., \& Oliveira, D. K. S. (2017). Percepções de usuários e profissionais da saúde da família sobre o Núcleo de Apoio à Saúde da Família. Saúde Debate, 41(115):1090-1101. https://doi.org/10.1590/0103-1104201711508.

Arce, V. A. R., \& Teixeira, C. F. (2017). Práticas de saúde e modelo de atenção no âmbito do Núcleo de Apoio à Saúde da Família em Salvador (BA). Saúde debate, 41(3):228-240. https://doi.org/10.1590/0103-11042017S317.

Azevedo, A. B., Pezzato, L. M., \& Mendes, R. (2017). Formação interdisciplinar em saúde e práticas coletivas. Saúde debate, 41(113):647-657. https://doi.org/10.1590/0103-1104201711323.

Bardin, L. (2016). Análise de Conteúdo. Edições 70.

Bispo Júnior, J. P., \& Moreira, D. C. (2018). Cuidado colaborativo entre os Núcleos de Apoio à Saúde da Família e as equipes apoiadas. Physis, 28(3):1-20. https://doi.org/10.1590/S0103-73312018280310.

Brasil (2008). Portaria $\mathrm{n}^{\mathrm{o}}$ 154, de 24 de janeiro de 2008. Credencia municípios conforme quantitativo e modalidade definidos, para receber o incentivo financeiro aos Núcleos de Apoio à Saúde da Família-Nasf. Diário Oficial da União; 25 jan. 2008. https://bvsms.saude.gov.br/bvs/saudelegis/gm/2008/prt0154_24_01_2008.html. 
Brasil (2012). Resolução n. 466, de 12 de dezembro de 2012. Aprova diretrizes e normas regulamentadoras de pesquisas envolvendo seres humanos (2012). Brasília, Diário Oficial da União; 12 dez. 2012. https://bvsms.saude.gov.br/bvs/saudelegis/cns/2013/res0466_12_12_2012.html.

Brasil (2014). Núcleo de Apoio à Saúde da Família - Volume 1: Ferramentas para a gestão e para o trabalho cotidiano. Brasília: Ministério da Saúde. https://bvsms.saude.gov.br/bvs/publicacoes/nucleo_apoio_saude_familia_cab39.pdf.

Brasil (2017a). Portaria $\mathrm{n}^{\circ}$ 2.436, de 21 de setembro de 2017. Aprova a Política Nacional de Atenção Básica, estabelecendo a revisão de diretrizes para a organização da Atenção Básica, no âmbito do Sistema Único de Saúde (SUS). Diário Oficial da União; 22 set. 2017 https://bvsms.saude.gov.br/bvs/saudelegis/gm/2017/prt2436_22_09_2017.html.

Brasil (2017b). Contribuições dos Núcleos de Apoio à Saúde da Família para a Atenção Nutricional. Brasília-DF: Ministério da Saúde. https://bvsms.saude.gov.br/bvs/publicacoes/contribuicoes_saude_familia_atencao_nutricional.pdf.

Campos, D. A., Vieira, M., \& Pires, R. O. M. (2017). Avaliação dos processos de trabalho dos nutricionistas dos núcleos de apoio à saúde da família do Meio Oeste de Santa Catarina. Sau. \& Transf. Soc., 8(1):84-97. http://incubadora.periodicos.ufsc.br/index.php/saudeetransformacao/article/view/4855/4973.

Campos, G. W. S., Figueiredo, M. D., Pereira Junior, N., \& Castro, C. P. (2014). A aplicação da metodologia Paideia no apoio institucional, no apoio matricial e na clínica ampliada. Interface, 18(1):983-995. https://doi.org/10.1590/1807-57622013.0324.

Castro, C. P., \& Campos, G. W. S. (2016). Apoio Matricial como articulador das relações interprofissionais entre serviços especializados e atenção primária à saúde. Physis, 26(2):455-481. https://doi.org/10.1590/S0103-73312016000200007.

Castro, C. P., Oliveira, M. M., Campos, G. W. S. (2016). Apoio Matricial no SUS Campinas: análise da consolidação de uma prática interprofissional na rede de saúde. Cienc Saude colet, 21 (5):1625-1636. https://doi.org/10.1590/1413-81232015215.19302015.

Costa, M., Peduzzi, M., Freire Filho, J. R., \& Silva, C. B. G. (2018). Educação interprofissional em saúde. Natal: SEDIS-UFRN. https://antigo.saude.gov.br/images/pdf/2018/dezembro/12/Educacao-Interprofissional-em-Saude.pdf.

Cynthia, M. B. N., Paulette, C. A., Fabiana, O. S. S., Luciana, C. A., \& Ide, G. D. G. (2018). Configurações do processo de trabalho em núcleos de apoio à saúde da família e o cuidado integral. Trab. Educ. saúde, 16(3), 1135-1156. https://doi.org/10.1590/1981-7746-sol00154.

Faria, L., Quaresma, M. A., Patiño, R. A., Siqueira, R., \& Lamego, G. (2018). Integração ensino-serviço-comunidade nos cenários de práticas na formação interdisciplinar em Saúde: uma experiência do Programa de Educação pelo Trabalho para a Saúde (PET-Saúde) no sul da Bahia, Brasil. Interface, 22(67):1257-1266. https://doi.org/10.1590/1807-57622017.0226.

Ferrer, M. L. P., Silva, A. S., Silva, J. R. K., \& Padula, R. S. (2015) Microrregulação do acesso à rede de atenção em fisioterapia: estratégias para a melhoria do fluxo de atendimento em um serviço de atenção secundária. Fisioter. Pesq., 22(3):223-230. https://doi.org/10.590/1809-2950/13038422032015.

Fittipaldi, A. L. M., Barros, D. C., \& Romano, V. F. (2017). Apoio matricial nas ações de alimentação e nutrição: Visão dos profissionais da Estratégia Saúde da Família. Physis, 27(3):793-811. https://doi.org/10.1590/S0103-73312017000300021.

Gil, A. C. (2008) Métodos e técnicas de pesquisas sociais. (6a ed.), Atlas.

Gonçalves, R. M. A., Lancman, S., Sznelwar, L. I., Cordone, N. G., \& Barros, J. O. (2015). Estudo do trabalho em Núcleos de Apoio à Saúde da Família (NASF), São Paulo, Brasil. Rev. bras. Saúde ocup., 40(131):59-74. https://doi.org/10.1590/0303-7657000078013.

Gozzi, A. P. N. F. (2017). A prática no Núcleo de Apoio à Saúde da Família (NASF): apoio matricial como inovação tecnológica em saúde (Tese de doutorado). São $\quad$ Carlos: Universidade $\quad$ Federal de https://repositorio.ufscar.br/bitstream/handle/ufscar/9940/GOZZI_Alana_2018.pdf?sequence=5\&isAllowed=y.

Hirdes, A. (2015). A perspectiva dos profissionais da Atenção Primária à Saúde sobre o apoio matricial em saúde mental. Cien saude colet, 20(2):371-382. https://doi.org/10.1590/1413-81232015202.11122014.

Instituto Brasileiro de Geografia e Estatística (2019). Cidade - Tauá. Panorama. https://cidades.ibge.gov.br/brasil/ce/taua/panorama.

Klein, A. P., \& d'Oliveira, A. F. P. L. (2017) O "cabo de força" da assistência: concepção e prática de psicólogos sobre o Apoio Matricial no Núcleo de Apoio à Saúde da Família. Cad Saude Publica, 33(1):1-10. https://doi.org/10.1590/0102-311X00158815.

Maffissoni, A. L., Silva, K. J., Vendruscolo, C., Trindadde, L. L., \& Matelski, F. K. (2018). Função matriciadora dos Núcleos Ampliados de Saúde da Família: uma revisão integrativa da literatura. Saúde debate, 42(119):1012-1023. https://doi.org/10.1590/0103-1104201811918.

Merhy, E. E., Baduy, R. S., Seixas, C. T., Almeida, D. E. S., \& Slomp Júnior, H. (2016) Avaliação compartilhada do cuidado em saúde: Surpreendendo o instituído nas redes. Hexis. https:/edisciplinas.usp.br/pluginfile.php/5798651/mod_resource/content/1/Avaliacao\%20compartilhada\%20do\%2 0cuidado\%20em\%20saude\%20vol2.pdf.

Minayo, M. C. S. (2006). O desafio do conhecimento. (9a ed.), HUCITEC.

Nascimento, A. G., \& Cordeiro, J. C. (2019). Núcleo ampliado de saúde da família e atenção básica: análise do processo de trabalho. Trab. educ. saúde, 17(2):1-20. https://doi.org/10.1590/1981-7746-sol00194.

Neto, J. D. A., Albuquerque I. M. N., Lira, G. V., \& Bosi, M. L. M. (2018). Aspectos restritivos à integralidade nos Núcleos de Apoio à Saúde da Família: o olhar dos stakeholders. Physis, 28(4):1-25. https://doi.org/10.1590/S0103-73312018280417.

Oliveira, M. M., \& Campos, G. W. S. (2017). Formação para o Apoio Matricial: percepção dos profissionais sobre processos de formação. Physis, 27(4):187206. https://doi.org/10.1590/S0103-73312017000200002. 
Research, Society and Development, v. 10, n. 16, e554101624102, 2021

(CC BY 4.0) | ISSN 2525-3409 | DOI: http://dx.doi.org/10.33448/rsd-v10i16.24102

Santos, D. S., Mishima, S. M., \& Merhy, E. E. (2018). Processo de trabalho na Estratégia de Saúde da Família: potencialidades da subjetividade do cuidado para reconfiguração do modelo de atenção. Cienc Saude Colet, 23(3):861-870. https://doi.org/10.1590/1413-81232018233.03102016.

Santos, F. C. R. (2018) Nutricionistas Dos Núcleos De Apoio a Saúde Da Família (Nasf) No Estado Do Ceará: perfil e atuação profissional (Dissertação de mestrado). Fortaleza: Universidade Estadual do Ceará. https://siduece.uece.br/siduece/trabalhoAcademicoPublico.jsf?id=83561.

Seixas, C. T., Baduy, R. S., Cruz, K. T., Bortoletto, M. S. S., Slomp Junior, H., \& Merhy, E. E. (2019). O vínculo como potência para a produção do cuidado em saúde: o que usuários-guia nos ensinam. Interface (Botucatu), 23:1-14. https://doi.org/10.1590/Interface.170627.

Souza, T. T. (2018) Núcleo Ampliado de Saúde da Família e Atenção Básica (NASF-AB). (2a ed.), UFSC. https://ares.unasus.gov.br/acervo/html/ARES/14868/1/Apostila_NASF-AB_Núcleo\%20Telessaúde\%20SC\%20UFSC.pdf.

Souza, T. T., Knabben, R. J., \& Calvo, M. C. M. (2017). Caracterização de núcleos de apoio à saúde da família e integração às equipes de saúde da família vinculadas. Rev. APS, 20(4):551-564. http://periodicos.ufjf.br/index.php/aps/article/view/16067/8307.

Spina, N., Martins, P. A., Vedovato, G. M., Laporte, A. S. C., Zangirolani, L. T. O., \& Medeiros, M. A. T. (2018). Nutricionistas na atenção primária no município de Santos: atuação e gestão da atenção nutricional. Demetra, 18(4):117-134. https://doi.org/10.12957/demetra.2018.30969.

Viana, M. M. O., \& Campos G. W. S. (2018). Formação Paideia para o Apoio Matricial: uma estratégia pedagógica centrada na reflexão sobre a prática. Cad. Saúde Pública, 34(8):1-15. https://doi.org/10.1590/0102-311X00123617. 